\title{
Use of an rRNA Internal Transcribed Spacer Region To Distinguish Phylogenetically Closely Related Species of the Genera Zygosaccharomyces and Torulaspora
}

\author{
S. A. JAMES, ${ }^{1 *}$ M. D. COLLINS, ${ }^{2}$ AND I. N. ROBERTS ${ }^{1}$ \\ National Collection of Yeast Cultures, BBSRC Institute of Food Research, Norwich Laboratory, Norwich Research \\ Park, Colney, Norwich NR4 7UA, ${ }^{1}$ and Department of Microbial Physiology, BBSRC Institute of Food Research, \\ Reading Laboratory, Earley Gate, Reading RG6 6BZ, ${ }^{2}$ United Kingdom
}

\begin{abstract}
Analyses of the sequences of the small-subunit (18S) rRNA gene and two internal transcribed spacers (ITSs), ITS1 and ITS2, revealed that members of the yeast genera Torulaspora and Zygosaccharomyces are phylogenetically intermixed. Despite some minor differences in 18S rRNA-, ITS1-, and ITS2-derived trees, in general the patterns of the relationships inferred from the three chronometers were in good agreement. The ITS sequences of Torulaspora and Zygosaccharomyces species exhibited far greater interspecies differences than the 18S rRNA sequences and were better than $18 S$ rRNA sequences for measuring close genealogical relationships. Despite the existence of interstrain ITS sequence variation in some species, it is possible to identify conserved regions in both ITSs that are useful in species differentiation.
\end{abstract}

Small-subunit rRNA sequencing is currently revolutionizing our knowledge about the natural relationships among bacteria (18). Small-subunit rRNA acts as a mosaic of different degrees of sequence conservation (from highly conserved to semiconserved to highly variable regions), which allows the molecule to measure distant evolutionary relationships, as well as closer evolutionary relationships. Compared with bacteria, there is relatively little information concerning yeast phylogeny based on this chronometer available (9). Although sequence analyses of small-subunit rRNA should make significant contributions to yeast systematics, the high overall level of conservation of the molecule may restrict its value in resolving the phylogeny of many yeast genera which exhibit smaller evolutionary depths than bacterial genera. The important food spoilage ascomycetous yeast genus Zygosaccharomyces is a case in point (7). Recent $18 \mathrm{~S}$ rRNA gene sequence analyses revealed that the genus Zygosaccharomyces is relatively shallow in evolutionary terms (it exhibits an intrageneric level of divergence of approximately $1.8 \%$ ) (7). Although the genus Zygosaccharomyces has been shown to consist of a number of subdivisions, the relatively high levels of sequence similarity observed between some species (e.g., Zygosaccharomyces bailii and Zygosaccharomyces bisporus) appear to limit the value of 18S rRNA for differentiating genotypically closely related species.

In this study we analyzed the sequence of the internal transcribed spacer (ITS) region as an alternative to 18S rRNA for delineating genealogically closely related yeasts. In yeasts, as well as in other eukarya, the ITS region is located between the $18 \mathrm{~S}$ and $28 \mathrm{~S}$ rRNA genes. The ITS region is subdivided into the ITS1 region, which separates the $18 \mathrm{~S}$ and 5.8S rRNA genes, and the ITS2 region, which is found between the $5.8 \mathrm{~S}$ and $28 \mathrm{~S}$ rRNA genes. In principle, ITSs are attractive chronometers for determining the relationships of genotypically closely related species, as they have higher rates of divergence than 18S rRNA genes. At one time it was thought that these higher rates occurred because the spacers have no function, but recent findings of van der Sande et al. (16) and Musters et

${ }^{*}$ Corresponding author. Phone: 44-1603-255000. Fax: 44-1603458414. Electronic mail address: NCYCNCFB@BBSRC.AC.UK al. (11) have shown that both ITS regions play a role in primary rRNA processing.

In this paper we describe the results of a comparison of the ITS1 and ITS2 sequences of members of the genus Zygosaccharomyces which showed that these sequences are useful for differentiating and delineating genealogically closely related species. In addition, we describe the phylogenetic intermixing of the genera Zygosaccharomyces and Torulaspora as determined by both $18 \mathrm{~S}$ rRNA and ITS sequence analyses.

\section{MATERIALS AND METHODS}

Yeast strains. The Zygosaccharomyces and Tonulaspora strains used in this study are shown in Table 1 . All of the strains were cultivated on YM plates $(0.3 \%$ yeast extract, $0.3 \%$ malt extract, $0.5 \%$ peptone, $1.0 \%$ glucose; $\mathrm{pH} 5.5)$ at $24^{\circ} \mathrm{C}$.

PCR amplification and PCR product purification. PCR amplification of the 18 SRNA gene was performed as described by James et al. (7). The entire ITS region was amplified by using primers pITS1 (5'-TCCGTAGGTGAACCT GCGG) and pITS4 (5'-TCCTCCGCTTATTGATATG), which were derived from conserved regions of the $18 \mathrm{~S}$ and $28 \mathrm{~S}$ rRNA genes, respectively (17). The amplified products were purified by using a Wizard PCR Preps DNA purification system (Promega, Southampton, United Kingdom) according to the manufacturer's instructions.

Direct PCR sequencing. Direct sequencing of the PCR-amplified 18S rRNA genes and ITSs was performed by using a Sequenase version 2.0 sequencing kit (United States Biochemical Corp., Cleveland, Ohio) as described by Hutson et al. (6). The primers used to sequence the 18S rRNA gene were the primers described by James et al. (7), and these primers generated approximately $97 \%$ of the 18S rRNA gene sequence for Torulaspora globosa and Torulaspora pretoriensis. To determine the sequences of the ITSs of the Zygosaccharomyces and Torulaspora strains shown in Table 1, we used two internal primers, primer pITS3 (5'-GCATCGATGAAGAACGCAG), which targeted a conserved region at approximately positions 31 to 49 of the $5.8 \mathrm{~S}$ rRNA gene, and primer p5.8Sr (5'-ATGACRCTCAAACAGGCAT), which targeted a conserved region at approximately positions 156 to 138 of the $5.8 \mathrm{~S}$ rRNA gene, in addition to the two external amplification primers, primers pITS1 and pITS4.

Sequence analysis. The $18 \mathrm{~S}$ rRNA gene sequences were aligned by using the multiple-sequence alignment program PILEUP (3) contained in the Genetics Computer Group software package (4), version 7.2 . The alignments were adjusted manually. The ITS sequences were subjected to a pairwise analysis. Hypervariability, including extensive insertions and deletions, precluded the use of multiple sequence alignment programs for the ITS data sets. Levels of sequence similarity were calculated, and derived evolutionary distance $\left(K_{\text {nuc }}\right)(5)$ values were used to produce unrooted phylogenetic trees by the neighbor-joining (14) and unweighted pair group with mathematical average (UPGMA) cluster analysis (15) methods. The confidence values of branches on the neighbor-joining tree were determined by performing a bootstrap analysis in which 500 replicates were used (2). 
TABLE 1. Yeast strains used and their ITS1 and ITS2 sequence accession numbers

\begin{tabular}{|c|c|c|c|}
\hline \multirow[b]{2}{*}{ Species $^{a}$} & \multirow[b]{2}{*}{ Strain $^{b}$} & \multicolumn{2}{|c|}{ EMBL accession no. } \\
\hline & & $\begin{array}{c}\text { ITS1 } \\
\text { sequence }\end{array}$ & $\begin{array}{c}\text { ITS2 } \\
\text { sequence }\end{array}$ \\
\hline T. delbrueckii (Lindner) Lindner & CBS $1146^{\mathrm{T} c}$ & Z48309 & Z48311 \\
\hline T. globosa (Klöcker) van der Walt \& Johannsen & NCYC $820^{\mathrm{T}}$ & Z48335 & Z48336 \\
\hline T. pretoriensis (van der Walt \& Tscheuschner) van der Walt \& Johannsen & NCYC $524^{\mathrm{T}}$ & $\mathrm{Z} 48310$ & Z48312 \\
\hline \multirow{7}{*}{ Z. bailii (Lindner) Guilliermond } & NCYC $1416^{\mathrm{T}}$ & $\mathrm{X} 84640$ & $\mathrm{X} 84641$ \\
\hline & NCYC 573 & $\mathrm{X} 84640$ & $\mathrm{X} 84641$ \\
\hline & NCYC 1515 & $\mathrm{X} 84640$ & X84641 \\
\hline & NCYC 417 & X87129 & $\mathrm{X} 87130$ \\
\hline & NCYC 464 & X87129 & $\mathrm{X} 87130$ \\
\hline & NCYC 1427 & $\mathrm{X} 84732$ & $\mathrm{X} 84733$ \\
\hline & NCYC 1766 & $\mathrm{X} 84732$ & $\mathrm{X} 84733$ \\
\hline \multirow[t]{4}{*}{ Z. bisporus Naganishi } & NCYC $1495^{\mathrm{T}}$ & X84642 & $\mathrm{X} 84643$ \\
\hline & NCYC 171 & X84642 & X84643 \\
\hline & NCYC 1496 & $\mathrm{X} 84642$ & X84643 \\
\hline & NCYC 1555 & X84642 & X84643 \\
\hline Z. cidri (Legakis) Yarrow & NCYC $1567^{\mathrm{T}}$ & X48347 & Z48361 \\
\hline Z. fermentati Naganishi & CBS $707^{\mathrm{T}}$ & Z48358 & $\mathrm{Z} 48362$ \\
\hline Z. florentinus Castelli ex Kudriavzev & CBS $746^{\mathrm{T}}$ & Z48348 & Z48363 \\
\hline Z. mellis Fabian \& Quinet & NCYC $2403^{\mathrm{T}}$ & Z48349 & Z48365 \\
\hline Z. microellipsoides (Osterwalder) Yarrow & CBS $427^{\mathrm{T}}$ & Z48350 & Z48364 \\
\hline Z. mrakii Capriotti (Osterwalder) Yarrow & CBS $4218^{\mathrm{T}}$ & Z48351 & Z48366 \\
\hline \multirow{4}{*}{ Z. rouxii (Boutroux) Yarrow } & NCYC $568^{\mathrm{T}}$ & $\mathrm{X} 84644$ & X84645 \\
\hline & NCYC 381 & X84644 & X84645 \\
\hline & NCYC 565 & X84644 & X84645 \\
\hline & NCYC 579 & X84644 & $\mathrm{X} 84645$ \\
\hline
\end{tabular}

${ }^{a}$ Species descriptions are given in reference 19 .

${ }^{b}$ Abbreviations: NCYC, National Collection of Yeast Cultures, Norwich, United Kingdom; CBS, Centraalbureau voor Schimmelcultures, Delft, The Netherlands. ${ }^{c} \mathrm{~T}=$ type strain.

Nucleotide sequence accession numbers. The nucleotide sequences of the $18 \mathrm{~S}$ rRNA genes of $T$. globosa and $T$. pretoriensis have been assigned EMBL accession numbers $\mathrm{X} 84639$ and $\mathrm{X} 84638$, respectively. The EMBL accession numbers for the 28 ITS sequences which we determined are shown in Table 1.

$\mathbf{A}$

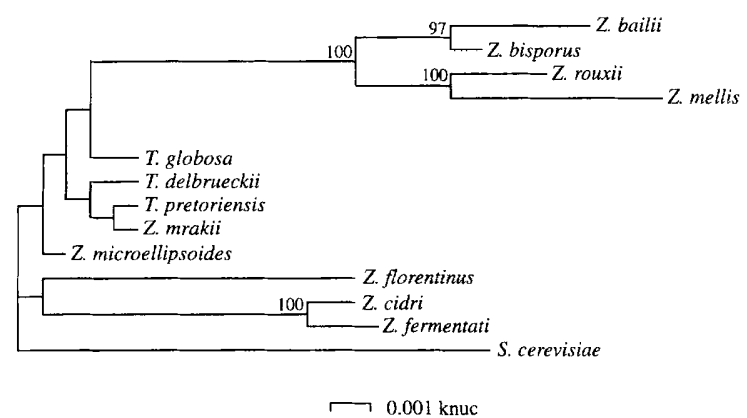

B

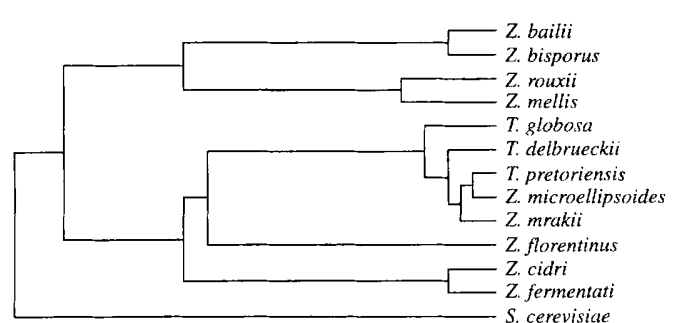

FIG. 1. Unrooted dendrograms showing the genetic relationships among Torulaspora and Zygosaccharomyces species. The dendrograms were constructed by the neighbor-joining method with bootstrap analysis (A) and the UPGMA cluster analysis method (B). Bar $=0.001 K_{\text {nuc. }}$ S. cerevisiae, Saccharomyces cerevisiae.

\section{RESULTS AND DISCUSSION}

$18 S$ rRNA gene sequence analysis. In a previous comparative 18S rRNA sequence study (7), Torulaspora delbrueckii, the type species of the genus Torulaspora, was found to be phylogenetically closely related to certain species belonging to the genus Zygosaccharomyces. Therefore, the 18S rRNA gene sequences of the other species of the genus Torulaspora, T. globosa and $T$. pretoriensis, were determined to elucidate the phylogenetic relationships of these organisms. Phylogenetic trees that were constructed by using both UPGMA cluster analysis (15) and neighbor-joining methods (14) and depict the relationship of species belonging to the genera Zygosaccharomyces and Torulaspora are shown in Fig. 1. High levels of sequence similarity were observed among the Torulaspora species (range, approximately 99.7 to $99.8 \%$ ), and the T. delbrueckii and $T$. pretoriensis sequences differed by only three bases. Similar high levels of sequence similarity have been observed for some Zygosaccharomyces species (e.g., Z. bailii and Z. bisporus) (7). Phylogenetically, the three Torulaspora species were closely related to Zygosaccharomyces species (range of levels of sequence similarity, approximately 98.4 to $99.9 \%$ ) and exhibited particularly close associations with Zygosaccharomyces mrakii and Zygosaccharomyces microellipsoides (levels of sequence similarity, 99.7 to $99.9 \%$ ). The results of our treeing analysis (Fig. 1) demonstrated that the Zygosaccharomyces and Torulaspora species are intermixed phylogenetically and that $Z$. microellipsoides, Z. mrakii, and the three Torulaspora species form a distinct subgroup that is separate from other Zygosaccharomyces species (Fig. 1). This significant association was more apparent on the UPGMA tree than on the neighborjoining tree; the latter exhibited a marked lack of ultrametricity.

ITS sequence analysis. The nucleotide sequences of ITS1 and ITS 2 regions of all of the strains shown in Table 1 were 


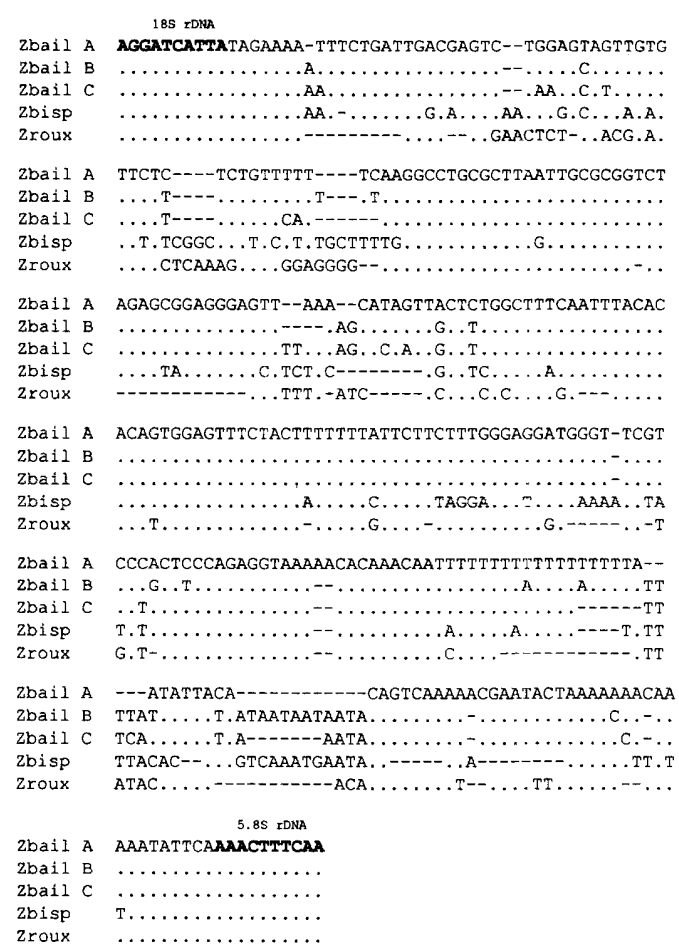

FIG. 2. Alignment of the ITS1 sequences of $Z$. bailii (Zbail), Z. bisporus (Zbisp), and Z. rouxii (Zroux), Zbail A, Z, bailii type A; Zbail B, Z, bailii type B; Zbail C, $Z$. bailii type $\mathrm{C}$.

determined by directly sequencing PCR-amplified fragments. Length polymorphisms were observed between species and in some cases between different strains of the same species for both ITS1 and ITS2. Within the genus Zygosaccharomyces, the size of ITS1 ranged from 166 to 292 bp (Zygosaccharomyces florentinus had the smallest ITS1 and $Z$. mrakii had the largest), while the size of ITS2 ranged from 173 to $303 \mathrm{bp}$ ( $Z$. florentinus had the smallest ITS2 and Zygosaccharomyces mellis had the largest). Within the genus Torulaspora, the size of ITS1 ranged from 292 to $313 \mathrm{bp}$ (T. pretoriensis had the smallest

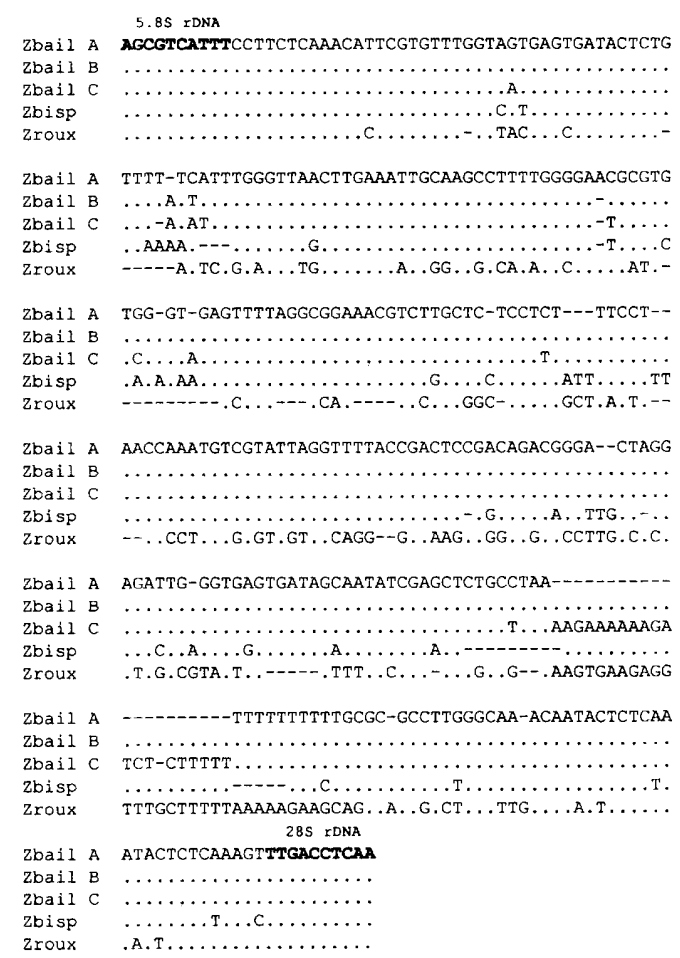

FIG. 3. Alignment of the ITS2 sequences of $Z$, bailii (Zbail), Z. bisponts (Zbisp), and Z. rouxii (Zroux). Zbail A, Z. bailii type A; Zbail B, Z. bailii type B; Zbail C, Z. bailii type C.

ITS1, and T. globosa had the largest), while the size of ITS2 varied by only a single base pair (ITS2 of $T$. delbrueckii and ITS2 of $T$. globosa were 258 bp long, and ITS2 of $T$. pretoriensis was 259 bp long).

The ITS1 and ITS2 sequences of Z. bailii, Z. bisporus, and $Z$ Zyosaccharomyces rouxii strains were aligned, and these sequences are shown in Fig. 2 and 3, respectively. Like Candida albicans (10), no interstrain sequence variation was observed for either $Z$. bisporus or $Z$. rouxii, although considerable se-

TABLE 2. Levels of ITS1 sequence similarity for Torulaspora and Zygosaccharomyces species

\begin{tabular}{|c|c|c|c|c|c|c|c|c|c|c|c|c|c|}
\hline \multirow[b]{2}{*}{ Taxon } & \multicolumn{13}{|c|}{$\%$ ITS1 sequence similarity } \\
\hline & 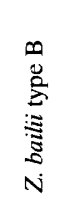 & 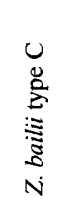 & 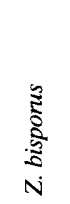 & $\begin{array}{l}\tilde{Z} \\
\mathbb{Z} \\
N\end{array}$ & 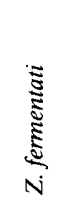 & 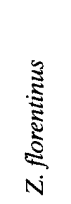 & 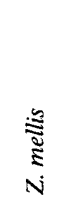 & 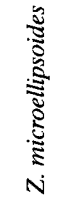 & 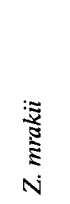 & 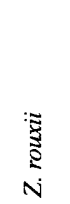 & 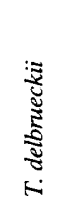 & 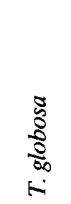 & 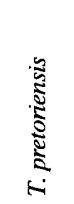 \\
\hline Z. bailii type A & 93.3 & 89.8 & 79.3 & 70.4 & 72.5 & 85.4 & 62.8 & 72.4 & 71.3 & 79.2 & 70.4 & 68.0 & 71.3 \\
\hline Z. bailii type B & & 92.9 & 81.9 & 70.8 & 72.1 & 84.0 & 60.3 & 70.3 & 69.7 & 79.8 & 68.9 & 63.9 & 65.9 \\
\hline Z. bailii type $\mathrm{C}$ & & & 82.0 & 70.8 & 73.4 & 84.1 & 59.9 & 69.5 & 68.8 & 80.6 & 74.2 & 67.3 & 69.2 \\
\hline Z. bisporus & & & & 71.1 & 71.2 & 79.4 & 66.0 & 68.2 & 66.6 & 76.7 & 64.2 & 66.9 & 67.3 \\
\hline Z. cidri & & & & & 95.2 & 68.7 & 72.8 & 77.8 & 76.8 & 62.8 & 78.0 & 79.6 & 76.8 \\
\hline Z. fermentati & & & & & & 72.1 & 73.1 & 77.7 & 77.5 & 60.9 & 80.5 & 79.9 & 77.1 \\
\hline Z. florentinus & & & & & & & 77.9 & 82.5 & 79.2 & 70.8 & 77.1 & 81.4 & 79.2 \\
\hline Z. mellis & & & & & & & & 67.1 & 65.9 & 76.6 & 66.0 & 69.6 & 66.0 \\
\hline Z. microellipsoides & & & & & & & & & 83.6 & 74.6 & 81.2 & 77.1 & 83.6 \\
\hline Z. mrakii & & & & & & & & & & 74.2 & 93.5 & 81.5 & 99.3 \\
\hline Z. rouxii & & & & & & & & & & & 76.8 & 77.3 & 75.0 \\
\hline T. delbrueckii & & & & & & & & & & & & 82.4 & 93.5 \\
\hline T. globosa & & & & & & & & & & & & & 81.8 \\
\hline
\end{tabular}


TABLE 3. Levels of ITS2 sequence similarity for Torulaspora and Zygosaccharomyces species

\begin{tabular}{|c|c|c|c|c|c|c|c|c|c|c|c|c|c|}
\hline \multirow[b]{2}{*}{ Taxon } & \multicolumn{13}{|c|}{$\%$ ITS2 sequence similarity } \\
\hline & 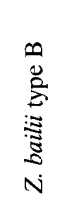 & 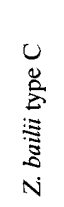 & 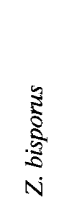 & $\begin{array}{l}\mathbf{5} \\
\mathrm{S} \\
\mathrm{N}\end{array}$ & 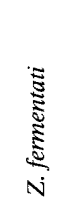 & 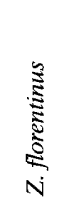 & $\begin{array}{c}\stackrel{\Xi}{\Xi} \\
\text { N } \\
N\end{array}$ & 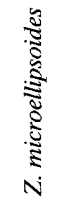 & 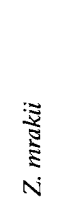 & 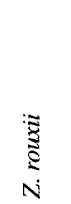 & 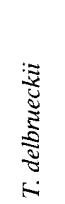 & 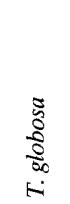 & 芯 \\
\hline Z. bailii type A & 98.8 & 96.1 & 87.0 & 75.8 & 75.0 & 81.1 & 68.1 & 72.4 & 72.2 & 56.0 & 72.1 & 72.4 & 71.8 \\
\hline Z. bailii type B & & 96.4 & 87.4 & 73.3 & 73.3 & 81.1 & 67.8 & 72.1 & 71.4 & 53.0 & 71.7 & 71.3 & 71.4 \\
\hline Z. bailii type $\mathrm{C}$ & & & 88.2 & 77.1 & 77.8 & 82.5 & 59.7 & 69.9 & 70.2 & 60.8 & 72.4 & 72.0 & 70.6 \\
\hline Z. bisporus & & & & 71.8 & 72.2 & 81.0 & 65.8 & 71.4 & 73.0 & 67.3 & 72.9 & 73.0 & 72.5 \\
\hline Z. cidri & & & & & 97.6 & 74.1 & 77.3 & 80.6 & 79.9 & 68.9 & 79.5 & 80.2 & 79.9 \\
\hline Z. fermentati & & & & & & 74.5 & 77.3 & 78.5 & 80.3 & 69.4 & 81.0 & 80.2 & 80.3 \\
\hline Z. florentinus & & & & & & & 82.9 & 81.2 & 79.1 & 80.2 & 79.1 & 77.9 & 79.1 \\
\hline Z. mellis & & & & & & & & 66.6 & 66.7 & 80.4 & 64.5 & 67.7 & 66.7 \\
\hline Z. microellipsoides & & & & & & & & & 89.6 & 54.4 & 88.8 & 88.8 & 89.6 \\
\hline Z. mrakii & & & & & & & & & & 56.6 & 97.3 & 93.4 & 100.0 \\
\hline Z. rouxii & & & & & & & & & & & 55.1 & 59.3 & 53.4 \\
\hline T. delbrieckii & & & & & & & & & & & & 94.6 & 96.9 \\
\hline T. globosa & & & & & & & & & & & & & 93.4 \\
\hline
\end{tabular}

quence variation was observed among $Z$. bailii strains. Within $Z$. bailii, three distinct ITS sequence types were identified; type A included strains NCYC 1416 (the type strain), NCYC 573, and NCYC 1515; type B included strains NCYC 1427 and NCYC 1766; and type C included strains NCYC 417 and NCYC 464. A pairwise analysis of the three Z. bailii ITS types revealed similarity values ranging from 89.8 to $93.3 \%$ for ITS1 and from 96.1 to $98.8 \%$ for ITS2. Despite the considerable intraspecific sequence variation in the ITSs of $Z$. bailii, we

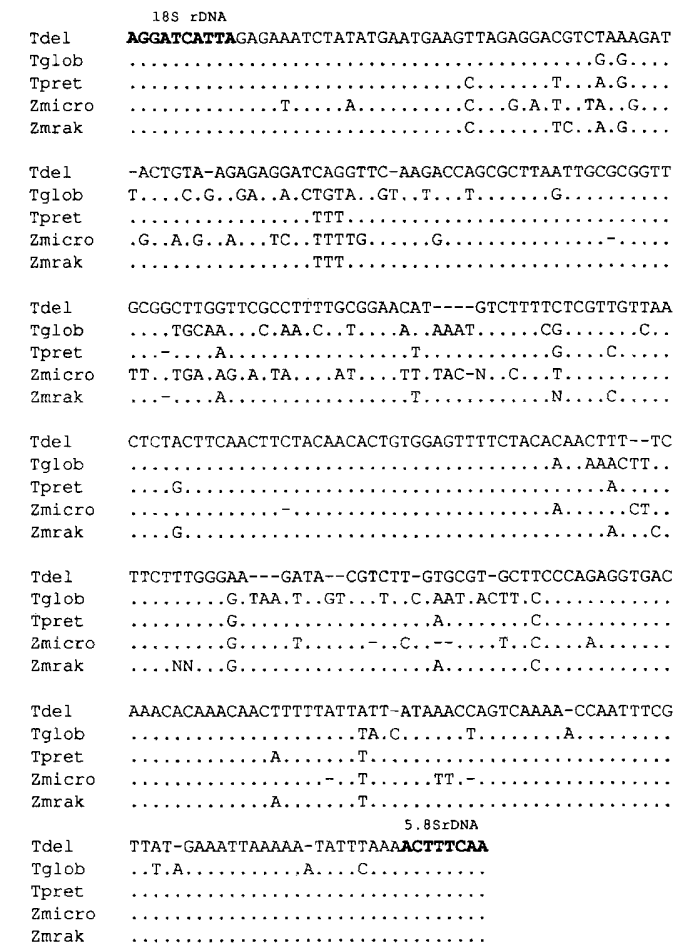

FIG. 4. Alignment of the ITS1 sequences of T. delbrueckii (Tdel), T. globosa (Tglob), T. pretoriensis (Tpret), Z. microellipsoides (Zmicro), and Z. mrakii (Zmrak). identified regions of homology in types $\mathrm{A}, \mathrm{B}$, and $\mathrm{C}$ that could be used to distinguish this species from $Z$. bisporus and $Z$. rouxii (Fig. 2 and 3 ). Alignments of the ITS1 and ITS2 regions of the three Torulaspora species, Z. microellipsoides, and $Z$. mrakii are shown in Fig. 4 and 5. A pairwise analysis revealed ranges of levels of sequence similarity of 77.1 to $99.3 \%$ for ITS1 and 88.8 to $100 \%$ for ITS2. For both spacers, the highest levels of sequence similarity were observed with $Z$. mrakii and T. pretoriensis $(99.3 \%$ for ITS1 and $100 \%$ for ITS2). Other very similar ITS sequences worthy of note are the sequences of Zygosaccharomyces cidri and Zygosaccharomyces fermentati (levels of sequence similarity for ITS1 and ITS2, 95.2 and

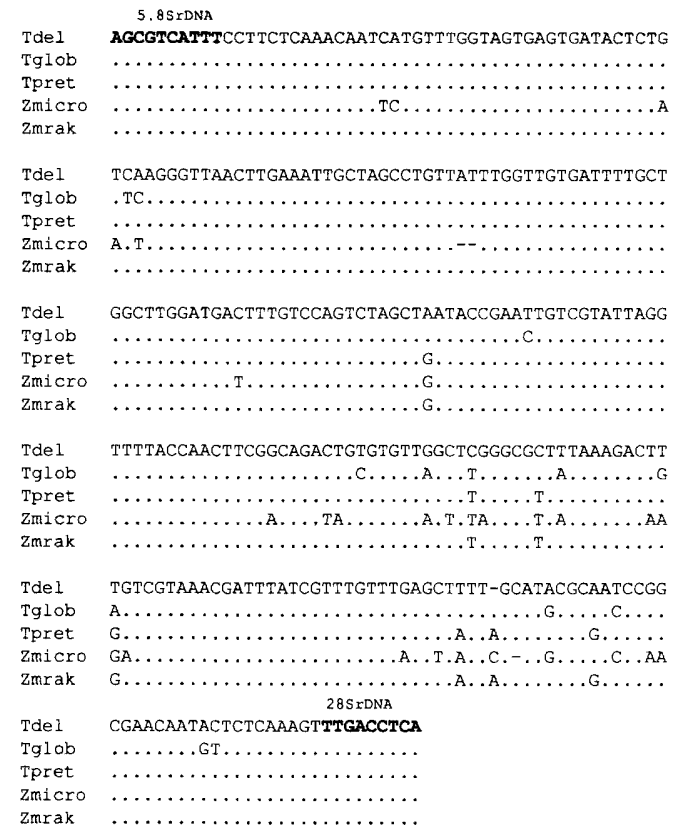

FIG. 5. Alignment of the ITS2 sequences of $T$, delbrueckii (Tdel), T. globosa (Tglob), T. pretoriensis (Tpret), Z. microellipsoides (Zmicro), and Z. mrakii (Zmrak). 

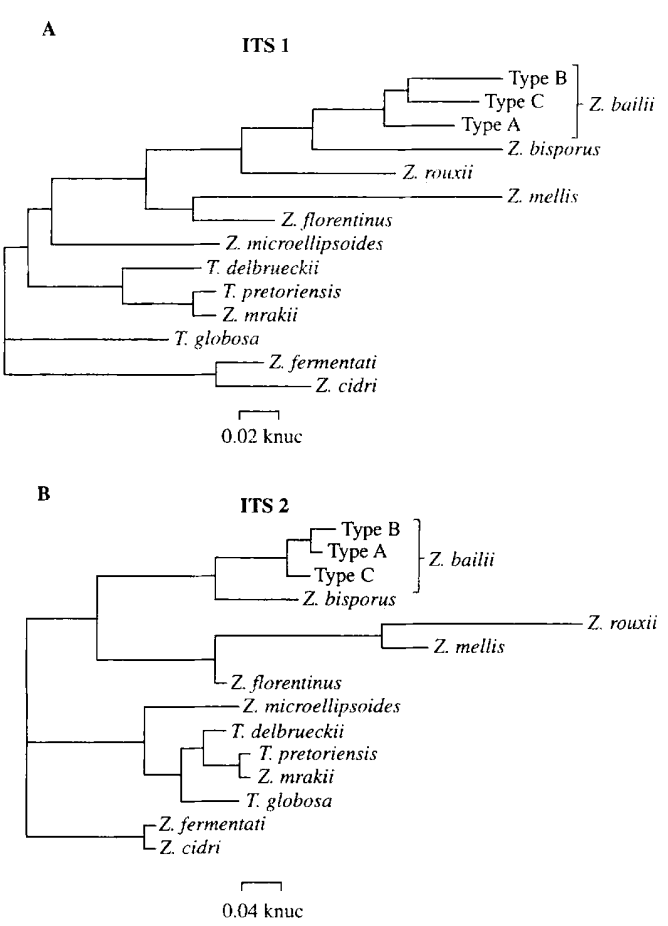

FIG. 6. Unrooted dendrograms constructed by the neighbor-joining method from ITS1 sequences (A) and ITS2 sequences (B).

$97.6 \%$, respectively) (Tables 2 and 3 ). Species belonging to the genus Zygosaccharomyces have been shown to be distinct as determined by nuclear DNA-DNA pairing (8). The presence of unique ITS sequences in all nine species of this genus is consistent with the genotypic distinctiveness of these organisms. The DNA-DNA pairing data for Torulaspora species are limited (13). However, the comparative species data that do exist are consistent with the ITS distinctiveness of these taxa.

Figure 6 shows the genealogical relationships of Zygosaccharomyces and Torulaspora species determined by using ITS1 and ITS2 sequences. Although ITS1 generally exhibited more sequence divergence than ITS2 (Tables 2 and 3), the tree topologies derived from the two data sets were for the most part in good agreement. For example, consistent with previously recognized phenotypic traits $(1,8,19), Z$. bailii (types A to C) and $Z$. bisporus formed a distinct subgroup, as did the species $Z$. cidri and $Z$. fermentati. Some differences in the pattern of the relationship between $Z$. mellis and $Z$. rouxii were evident on the trees derived from $18 \mathrm{~S}$ rRNA and ITS sequences. The $18 \mathrm{~S}$ rRNA data indicated that $Z$. mellis and $Z$. rouxii are closely related to each other and significantly related to the $Z$. bailii-Z. bisporus subgroup (7). On the ITS1-derived tree, $Z$. mellis did not exhibit a close genealogical relationship to $Z$. rouxii (Fig. $6 \mathrm{~A})$, although these species were closely associated on the ITS2-derived tree, in accordance with the $18 \mathrm{~S}$ rRNA data. The close genealogical relationship among $T$. delbrueckii, $T$. pretoriensis, and $Z$. mrakii inferred from the comparative $18 \mathrm{~S}$ rRNA analysis (Fig. 1) was confirmed by both the ITS1 tree and the ITS2 tree (Fig. 6). It is clear that T. delbrueckii, T. globosa, $T$. pretoriensis, $Z$. mrakii, and $Z$. microellipsoides form a tight phylogenetic subgroup and should be placed in the same genus. These results are consistent with the phenotypic similarities of these species $(1,19)$. Although it is tempting to suggest that these findings support reclassification of $Z$. microellipsoides and $Z$. mrakii in the genus Torulaspora, we believe that such a move would be premature. Several other yeast genera may also be phylogenetically intermixed with the genus Zygosaccharomyces (e.g., the genera Saccharomyces [7] and Kluyveromyces [unpublished data]). Consequently, additional genealogical comparisons will be necessary before any phylogenetic rationale for subdividing this group of highly related ascomycetous yeasts can be put forward with confidence.

Despite some minor incongruencies in the $18 \mathrm{~S}$ rRNA, ITS1, and ITS2 data, in general the patterns of relationships derived from the three sequence data sets were in good agreement. Although 18S rRNA is an immensely powerful chronometer for elucidating phylogenetic relationships, its high overall level of sequence conservation seems to limit its value for measuring and resolving close relationships. It is evident from our data that ITS sequences exhibit far greater interspecies sequence divergence and because of this are superior to 18S rRNA for measuring very close genealogical relationships (i.e., relationships of closely related species or species groups). However, the hypervariability of ITSs and the associated alignment problems (increasing noise and error) preclude the use of ITS for assessing more distant relationships. Hence, whenever possible, it is advisable to combine information from both types of data sets when close phylogenetic relationships are studied. It is pertinent to note that despite considerable interstrain ITS sequence variation in some species (e.g., Z. bailii types A, B, and C) (12), it is clearly possible to identify conserved regions ("core sequences") in both spacers which can be used for species delineation. Such regions could also be invaluable for rapid species identification with probes.

\section{ACKNOWLEDGMENT}

We are grateful to the EEC Agricultural Industrial Research Programme (contract CT93-0830) for support.

\section{REFERENCES}

1. Barnett, J. A., R. W. Payne, and D. Yarrow. 1990. Yeasts: characteristics and identification, 2nd ed. Cambridge University Press, Cambridge.

2. Felsenstein, J. 1993. PHYLIP: phylogenetic inference package, version 3.5 . University of Washington, Seattle.

3. Feng, D. F., and R. F. Doolittlte. 1987. Progressive sequence alignment as a prerequisite to correct phylogenetic trees. J. Mol. Evol. 35:351-360.

4. Genetics Computer Group. 1991. Program manual for the GCG package, version 7. Genetics Computer Group, Madison, Wis.

5. Hori, H., and S. Osawa. 1979. Evolutionary change in 5S RNA secondary structure and a phylogenetic tree of 54 5S RNA species. Proc. Natl. Acad. Sci. USA 76:381-385.

6. Hutson, R. A., D. E. Thompson, and M. D. Collins. 1993. Genetic interrelationships of saccharolytic Clostridium botulinum types B, E and F and related clostridia as revealed by small-subunit rRNA gene sequences. FEMS Microbiol. Lett. 108:103-110.

7. James, S. A., M. D. Collins, and I. N. Roberts. 1994. Genetic interrelationship among species of the genus Zygosaccharomyces as revealed by smallsubunit rRNA gene sequences. Yeast 10:871-881.

8. Kurtzman, C. P. 1990 . DNA relatedness among species of the genus $Z y$ gosaccharomyces. Yeast 6:213-219.

9. Kurtzman, C. P., and C. J. Robnett. 1991. Phylogenetic relationships among species of Saccharomyces, Schizosaccharomyces, Debaryomyces and Schwanniomyces determined from partial ribosomal RNA sequences. Yeast 7:61-72.

10. Lott, T. J., R. J. Kuykendall, and E. Reiss. 1993. Nucleotide sequence analysis of the 5.8S rDNA and adjacent ITS2 region of Candida albicans and related species. Yeast 9:1199-1206.

11. Musters, W., K. Boon, C. A. F. M. van der Sande, H. van Heerikhuizen, and R. J. Planta. 1990. Functional analysis of transcribed spacers of yeast ribosomal DNA. EMBO J. 9:3989-3996.

12. O'Donnell, K. 1992. Ribosomal DNA internal transcribed spacers are highly divergent in the phytopathogenic ascomycete Fusarium sambicum (Gibberella pulicaris). Curr. Genet. 22:213-220.

13. Price, C. W., G. B. Fuson, and H. J. Phaff. 1978. Genome comparison in yeast systematics: delimitation within the genera Schwanniomyces, Saccharomyces, Debaryomyces and Pichia. Microbiol. Rev. 42:161-193.

14. Saitou, N., and M. Nei. 1987. The neighbor-joining method: a new method for reconstructing phylogenetic trees. Mol. Biol. Evol. 4:406-425.

15. Swofford, D. L., and G. Olsen. 1990. Phylogenetic reconstruction, p. 411-501. 
In D. M. Hillis and C. Moritz (ed.), Molecular systematics. Sinauer Associates, Inc., Publishers, Sunderland, Mass.

16. van der Sande, C. A. F. M., M. Kwa, R. W. van Nues, H. van Heerikhuizen, H. A. Raue, and R. J. Planta. 1992. Functional analysis of internal transcribed spacer 2 of Saccharomyces cerevisiae ribosomal DNA. J. Mol. Biol. 223:899-910.

17. White, T. J., T. D. Bruns, S. Lee, and J. W. Taylor. 1990. Amplification and direct sequencing of fungal ribosomal RNA genes for phylogenetics, p. 315-322. In M. Innis, D. H. Gelfand, J. J. Sninsky, and T. J. White (ed.), PCR protocols. Academic Press, San Diego, Calif.

18. Woese, C. R. 1987. Bacterial evolution. Microbiol. Rev. 51:221-271.

19. Yarrow, D. 1984. Zygosaccharomyces Barker, p. 449-465. In N. J. W. Kregervan Rij (ed.), The yeasts: a taxonomic study, 3rd ed. Elsevier Science Publishers, Amsterdam. 